\title{
The Performance of Active Coated Nanoparticles Based on Quantum-Dot Gain Media
}

\author{
Sawyer D. Campbell ${ }^{1}$ and Richard W. Ziolkowski ${ }^{1,2}$ \\ ${ }^{1}$ College of Optical Sciences, University of Arizona, Tucson, AZ 85721, USA \\ ${ }^{2}$ Department of Electrical and Computer Engineering, University of Arizona, Tucson, AZ 85721, USA
}

Correspondence should be addressed to Richard W. Ziolkowski, ziolkowski@ece.arizona.edu

Received 25 June 2012; Revised 21 August 2012; Accepted 25 August 2012

Academic Editor: Pavel A. Belov

Copyright ( $) 2012$ S. D. Campbell and R. W. Ziolkowski. This is an open access article distributed under the Creative Commons Attribution License, which permits unrestricted use, distribution, and reproduction in any medium, provided the original work is properly cited.

\begin{abstract}
Quantum-dots (QDs) provide an exciting option for the gain media incorporated in active coated nanoparticles (CNPs) because they possess large gain coefficients resulting from their extreme confinement effects. The optical properties of core/shell QDs can be tuned by changing the relative size of the core/shell, that is, by effectively changing its band gap structure. Similarly, the resonance of a CNP can be adjusted by changing the relative sizes of its layers. It is demonstrated here that by optimally locating the QDs inside a resonant CNP structure it is possible to greatly enhance the intrinsic amplifying behavior of the combined QD-CNP system.
\end{abstract}

\section{Introduction}

The active coated nanoparticle (CNP) designs studied previously considered active silica cores coated with either Ag or Au shells (depending on the wavelength region of interest) [1-3] or the corresponding "inside-out" (IO) designs, that is, metallic cores covered with active silica coatings [2, 4]. In general, these designs used a simple gain model that did not include the dispersion behavior typical of a physical, active medium. These nanoamplifier designs were predicted to require a gain value of $10^{4}-10^{5} \mathrm{~cm}^{-1}$. This is achievable with quantum dots (QDs); and, consequently, they have been a preferred choice for some discussions on the physical realizations of these nanoamplifiers [3]. QDs offer an alternative, more robust option to dye-based gain media, which are susceptible to bleaching. Nonetheless, the active IO-CNP has been experimentally verified with a dye impregnated silica coating [4]. Moreover, it and related studies $[5,6]$ suggest that the strong localized field and large cross-section effects associated with these plasmonic nanostructures reduce the model-based large gain values to more practical ones. We demonstrate here that QDs, which can be obtained commercially and could be integrated with the CNPs, do in fact represent an exciting practical option for a variety of active CNP designs. Moreover, it is established that the number of QDs needed for a successful active CNP design is considerably smaller than anticipated.

\section{Quantum-Dot Gain Model}

The effective permittivity of a core-shell QD has been modeled by Holmström et al. and is applicable for core-shell QDs in the strong confinement regime [7]. This model is motivated by Maxwell-Garnett effective medium theory and is given by the following:

$$
\epsilon_{\mathrm{QD}, \mathrm{Lor}}^{\mathrm{CS}}(\omega)=\epsilon_{b}^{\mathrm{CS}}+\frac{2}{V_{\mathrm{QD}}^{\mathrm{CS}}}\left(f_{c}-f_{v}\right) \frac{e^{2} f /\left(m_{0} \epsilon_{0}\right)}{\omega^{2}-\omega_{0}^{2}+i 2 \omega \gamma},
$$

where $V_{\mathrm{QD}}^{\mathrm{CS}}=(4 \pi / 3) R_{2}^{3}$ is the volume of the entire coreshell structure; $f$ is the oscillator strength; $f_{c}$ and $f_{v}$ are, respectively, the conduction and valence band carrier distribution functions; $\gamma$ is a dampening term associated with the resonance width. The model assumes that a pump signal at a frequency higher than the resonance frequency, $f_{\text {res }}=\omega_{0} / 2 \pi$, creates the difference between the conductionand valence-band populations: $\left(f_{c}-f_{v}\right)$. However, because this population difference term is effectively related to the pump strength, it takes on values between 0 and 1 . It will be termed the gain scale. It supplies the model with the ability 
to represent a desired gain value at the resonance frequency. The core-shell background dielectric constant $\epsilon_{b}^{\mathrm{CS}}$ is given by the following:

$$
\epsilon_{b}^{\mathrm{CS}}=\epsilon_{s}+3 f_{\text {core }} \epsilon_{s} \frac{\epsilon_{c}-\epsilon_{s}}{\epsilon_{c}+2 \epsilon_{s}-f_{\text {core }}\left(\epsilon_{c}-\epsilon_{s}\right)}
$$

where $f_{\text {core }}$ is ratio of the core volume to the entire QD volume, and $\epsilon_{c}$ and $\epsilon_{s}$ are the bulk dielectric constants of the core and shell, respectively. The model was originally applied to an InAs/GaAs QD which operated at a bandgap energy of $0.8 \mathrm{eV}(\lambda=1550 \mathrm{~nm})$. Because of the desire to conduct prototype experiments to demonstrate their potential nanosensor applications, we have extended it here to commercially available QD structures.

Many different types of quantum dots are now available commercially in the frequency range of interest. For instance, Sigma-Aldrich produces a range of $\mathrm{CdSe} / \mathrm{ZnS}$ core-shell quantum dots for use in the visible and near-infrared parts of the spectrum. The published properties of some of their CdSe/ZnS core-shell QDs are given in Table 1 [8]; they show a considerable range of available gain values and related optical extinction coefficients, $g=-2\left(\omega_{0} / c\right) \kappa, c$ being the vacuum speed of light.

Their $560 \mathrm{~nm}$ emitting QD, whose diameter is $6.0 \mathrm{~nm}$ was chosen for our study; it has a gain value which is close to those used in our previous studies [6]. Moreover, its core-shell configuration conforms nicely to our analytical simulation approach. The indexes of refraction for CdSe and $\mathrm{ZnS}$ at visible wavelengths are approximately 2.5 and 2.41 , respectively $[9,10]$. These values, along with the published QD material properties, were used to calculate the wavelength-dependent effective permittivity and, hence, the index of refraction of a QD layer. Figure 1 depicts the wavelength dependence of the index of refraction for the commercially available Sigma-Aldrich $560 \mathrm{~nm}$ QD based on the theoretical model given by (1)-(2). It will be denoted as "SA-560 QD" in the following discussions. The oscillator strength $f$ and the damping term $\gamma$ were chosen, respectively, to be 1.9185 and $5.0 \mathrm{meV}$ to match the corresponding peak gain value given in Table 1. Consequently, when the gain scale value equals one, the model recovers that peak gain value. As Table 1 shows, the observed QD resonance strength increases as the QD size becomes larger. As a consequence, its dispersion properties are quite large over the frequency band of interest. Moreover, the active CNP's resonant linewidth, as would be expected for a core-shell amplifier, is quite narrow [1]. While the available gain bandwidth for these QDs is wider than the active CNP resonance, both of these properties nevertheless present added degrees of difficulty in the design and fabrication of any QD-CNP structure. The center frequency of the CNP resonance is rather sensitive to the material properties of its layers and to their respective radii. To achieve the desired enhanced responses, the final configuration must provide a significant overlap of the CNP and QD resonances.

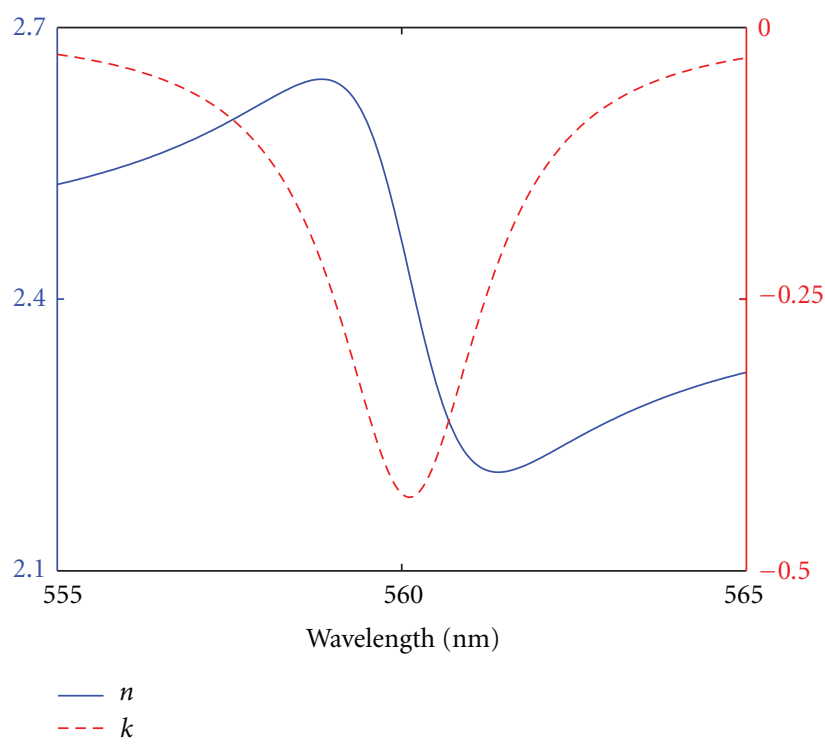

Figure 1: Behavior of the real, $n$, and imaginary, $\kappa$, parts of the index of refraction of the SA-560 QD as a function of the wavelength.

TAble 1: Physical properties of commercially available SigmaAldrich core-shell quantum dots.

\begin{tabular}{lcccc}
\hline $\begin{array}{l}\text { Emission } \\
\text { wavelength } \\
(\mathrm{nm})\end{array}$ & $g \times 10^{5}\left(\mathrm{~cm}^{-1}\right)$ & $\kappa$ & $\begin{array}{c}\text { Core } \\
\text { diameter } \\
(\mathrm{nm})\end{array}$ & $\begin{array}{c}\text { QD } \\
\text { diameter } \\
(\mathrm{nm})\end{array}$ \\
\hline 510 & 0.045 & -0.018 & 3.0 & 4.9 \\
530 & 0.065 & -0.027 & 3.3 & 5.2 \\
560 & 0.97 & -0.432 & 3.4 & 6.0 \\
590 & 1.6 & -0.751 & 4.0 & 6.2 \\
610 & 4.90 & -2.379 & 5.2 & 7.7 \\
640 & 5.90 & -3.005 & 6.3 & 8.6 \\
\hline
\end{tabular}

\section{Active CNP Designs}

The previously studied CNP and corresponding IO designs possess strong resonant behaviors $[4,6]$ and serve as the basis for the QD-augmented designs reported here. In particular, we replace the active silica region in the previous designs with an equivalent cluster of QDs, which we treat as a continuous region whose thickness is determined by the number of SA-560 QDs that could fit radially into the layer and whose permittivity is specified by (1) and (2). We used our previously-developed Mie theory CNP models $[1,3,6,11]$ to calculate the behavior of the proposed QD-augmented CNP designs. Silver was selected for the metal; it was modeled with a size- and wavelength-dependent permittivity [1] that recovers the known measured results [12]. As noted above, a background pump signal is assumed to create the necessary population difference to achieve the gain value specified by the manufacturer and recovered by the QD permittivity model, (1). 


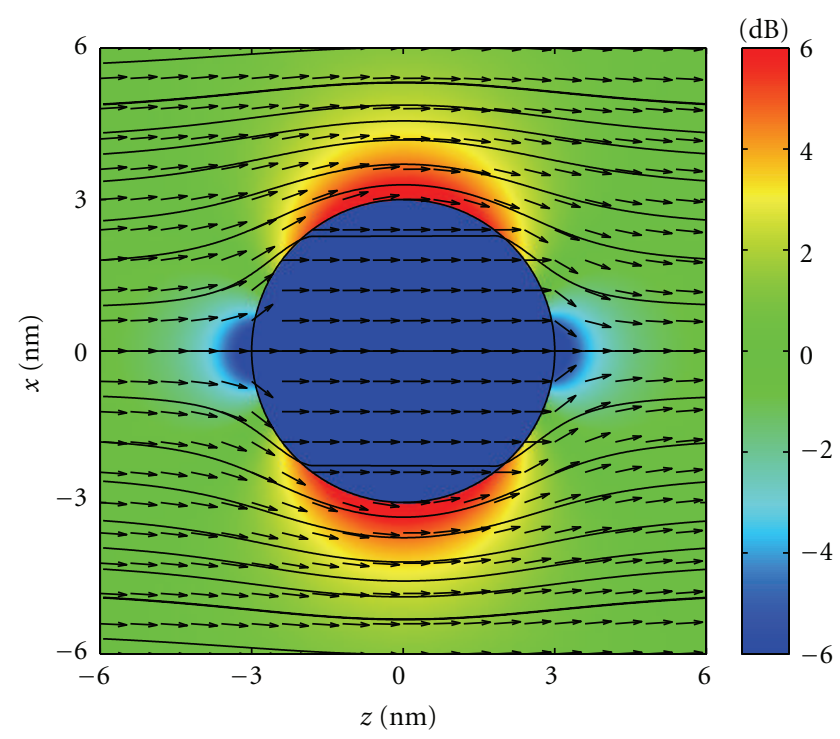

FIgURE 2: Contour plots of the electric field distribution along with the normalized total Poynting's vector field for a single SA-560 QD.

While a QD possesses amplifying behavior by itself, it only weakly couples to an incident field, which leads to a weak response. Figure 2 shows the electric field distribution and the corresponding Poynting's vector field for the SA560 QD when it is excited by an incident plane wave at its resonance frequency. The streamlines of the Poynting's vector field flow around the QD, indicating a weak interaction of the incident field with it.

On the other hand, if the QD is simply coated with a metal, the coupling to and the localization of the incident field energy in the gain region can be improved substantially and the resulting resonant plasmonic behavior will significantly enhance its response. We have found that while a single SA-560 QD, when coated with an Ag shell, can be designed to have a strong resonance, it does not have enough gain to overcome the intrinsic losses associated with that shell. On the other hand, slightly larger CNPs that contain several QDs generally exhibit stronger light-matter interactions with (i.e., have a stronger coupling to) the incident field and, hence, have larger resonant responses. A multilayered cluster of QDs can be employed in the core and if the shell is properly designed, the resulting multilayer gainaugmented core-shell system will exhibit a super-resonance state [1] when its gain value is large enough to overcome its intrinsic losses. Figure 3 shows the electric field distribution for a core size equivalent to the radius of "two" QD layers $(9 \mathrm{~nm})$, that is, a QD in the center and one full layer of dots surrounding it, when coated with a $3 \mathrm{~nm} \mathrm{Ag}$ shell. While the QD core region is treated as a continuous medium in the simulation, we have included circles that are overlayed in the core to represent the size of the individual QDs relative to the entire structure.

There is a significant enhancement in the electric field amplitude when compared to the single bare SA-560 QD seen in Figure 2. This enhancement effect can be increased with up to three layers of QDs. However, we found that it

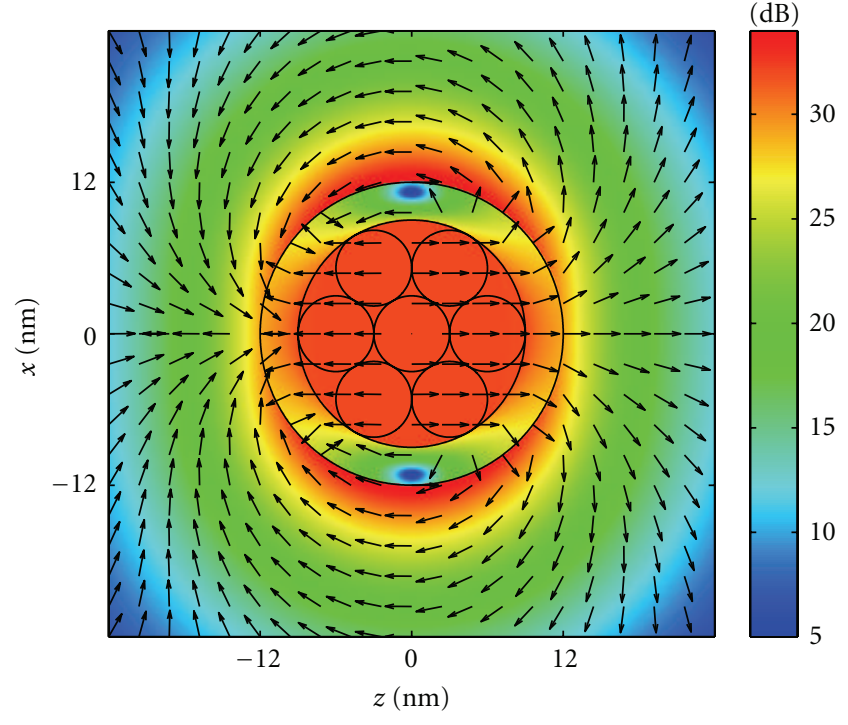

FIGURE 3: Contour plots of the electric field distribution along with the normalized total Poynting's vector field for a set of SA-560 QDs encapsulated in a $3.0 \mathrm{~nm}$ thick silver shell having a $12.0 \mathrm{~nm}$ exterior radius.

begins to fall off with four- and more layered cores because the gain is so large it significantly detunes the overlap of the QD and structural resonances. On the other hand, by placing the active region more strategically, as suggested by previous 3-layer designs [6], one can dramatically improve the active CNP performance while decreasing the number of QDs required.

The two designs whose performance characteristics are noticeably the best are the $\mathrm{Ag} / \mathrm{QD} / \mathrm{SiO}_{2}$ (called the QD-CNP) and $\mathrm{QD} / \mathrm{Ag} / \mathrm{SiO}_{2}$ (called the IO-CNP) configurations. Both are three-layer designs consisting of the same materials, but differ in the placement of the QD layer. The QD layer of the QD-CNP design lies between the Ag shell and the $\mathrm{SiO}_{2}$ core, while the QD layer of the IO-CNP is the outermost layer. The core and shell sizes were adjustable parameters; the QD size was fixed by the manufacturer's specifications. Both of the resulting optimized designs feature a $17.5 \mathrm{~nm}$ radius core and a $6 \mathrm{~nm}$ thick QD layer. For both cases, a single layer of QDs is sufficient to achieve a very large resonant response. The optimized thickness of the Ag shell in the QD-CNP design was $6.2 \mathrm{~nm}$ (29.7 nm total radius); it was $6.3 \mathrm{~nm}$ thick ( $29.8 \mathrm{~nm}$ total radius) for the IO-CNP design. We note that other three layer designs featuring a single QD inside a metal coated dielectric core/shell structure have been proposed [13].

However, we have found that placing the active material near the shell boundary can greatly increase the performance of the active CNP. In fact, while we have studied designs with the active region near, but separated from the shell, we have seen that the optimal location of the active region is directly adjacent to the shell. While it is known that placing the active region next to a metal will enhance the nonradiative decay rate of the active material, that is, it will quench the emission rate, recent theoretical and experimental studies have shown 


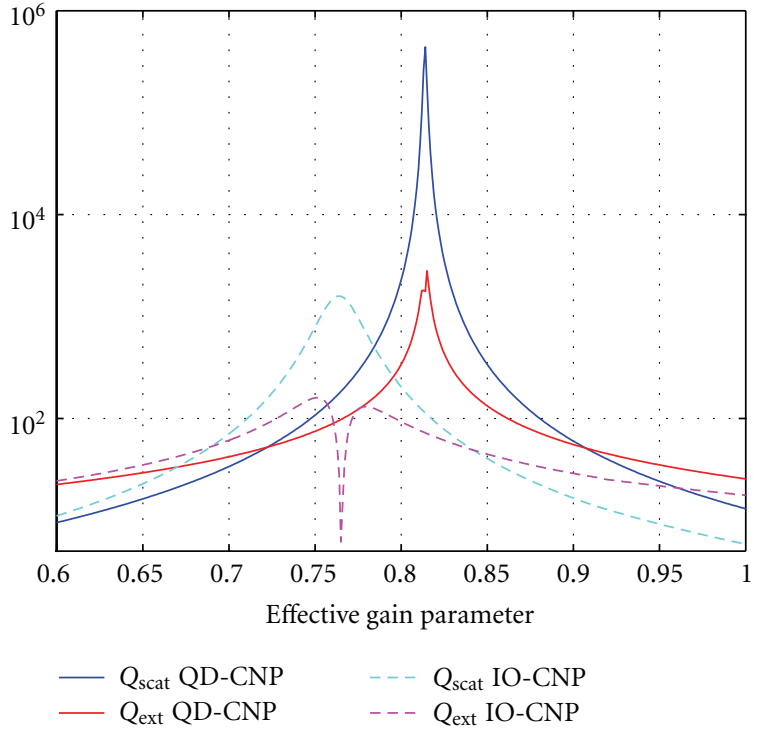

FIGURE 4: Scattering and extinction efficiencies for the optimized QD-CNP and the IO-CNP configurations.

that plasmonic-related effects can actually significantly enhance the radiative decay rates [14-20]. In particular, by properly designing a multi-layer structure to retain the unique optical and electronic properties of the QD and CNP and to provide a much larger density of radiating states to which the emissions can couple, one can produce active CNPs with very efficient florescence behaviors, especially for materials with high internal quantum efficiencies which QDs possess. Even though the metal layer is thin and the QDs are not, which would lead to lower quenching effects in any event, designs exhibiting the large radiated power enhancements associated with a super-resonance state [1] correspond to ones that foster enhanced radiative decays. Thus, the proposed optimized QD-CNP and IO-CNP designs avoid quenching and significantly outperform the single QD, twolayer version. The scattering efficiency and the absolute value of the extinction efficiency for these designs are plotted against the gain scale factor in Figure 4 . We note that the optimized configurations require less than the peak gain value to achieve their resonant states, which would be quite advantageous in practice when fabrication and experimental tolerances tend to negatively impact the ideal component performance characteristics. Also note that the extinction efficiency achieves a negative value on the larger gain factor value (right) side of the resonant peaks of the scattering efficiency. (we note that this feature appears in the $\left|Q_{\text {ext }}\right|$ curves as the sharp nulls since the absolute value is being plotted). Recall that when the extinction efficiency becomes negative, it means the losses are overcome and the incident field is amplified. One immediately observes that the scattering and extinction behaviors of the QD-CNP design are several orders of magnitude larger than those of the IO-CNP.

The resonant CNP-based designs have cross sections much larger than their physical size [6]; this behavior impacts the actual, rather than the model-based gain values needed for their operation. Figure 5 shows the power flow

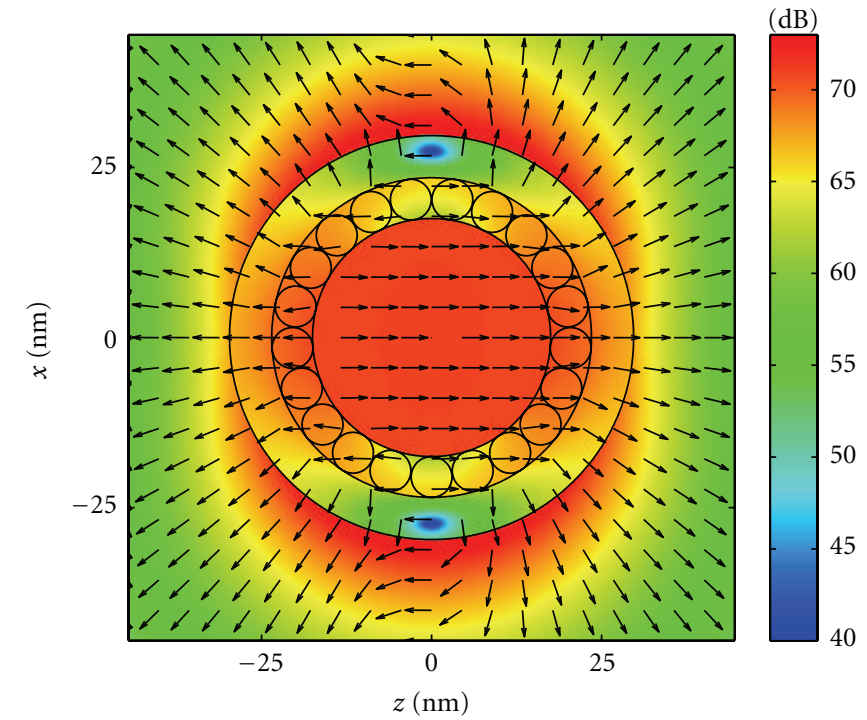

(a) QD-CNP

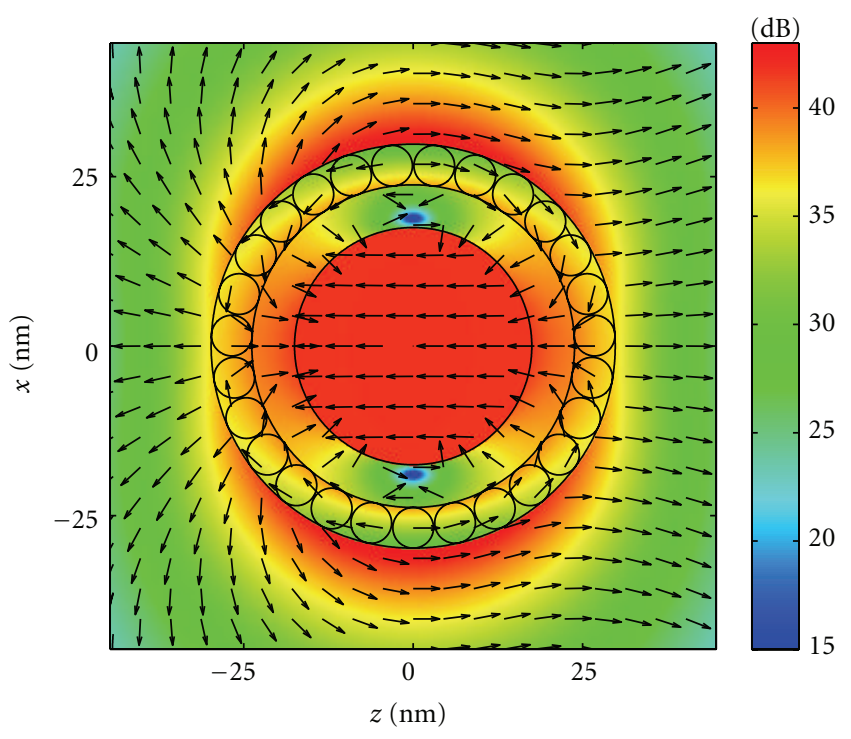

(b) IO-CNP

FIGURE 5: Contour plots of the electric field distribution along with the normalized total Poynting's vector field for the QD-CNP and IO-CNP designs.

behavior of the total Poynting's vector field for both designs when the incident plane wave has a $560 \mathrm{~nm}$ wavelength. The QDs are drawn as circles representing their actual size with respect to the size of the structure. In both the QD-CNP and IO-CNP cases, these structures produce a strong overall dipole resonance in their exteriors. However, it is clear that power is flowing more strongly outward from the QD layer in the QD-CNP structure than it is in the IO-CNP one (note that the minimum level in Figure 5(a) is the maximum one in Figure 5(b)), further emphasizing the large scattering cross section differences shown in Figure 4.

Although the gain region in the three-layer QD-CNP and IO-CNP designs is only a single layer of QDs, it has a volume equivalent to 282 and 481 individual dots, respectively. 


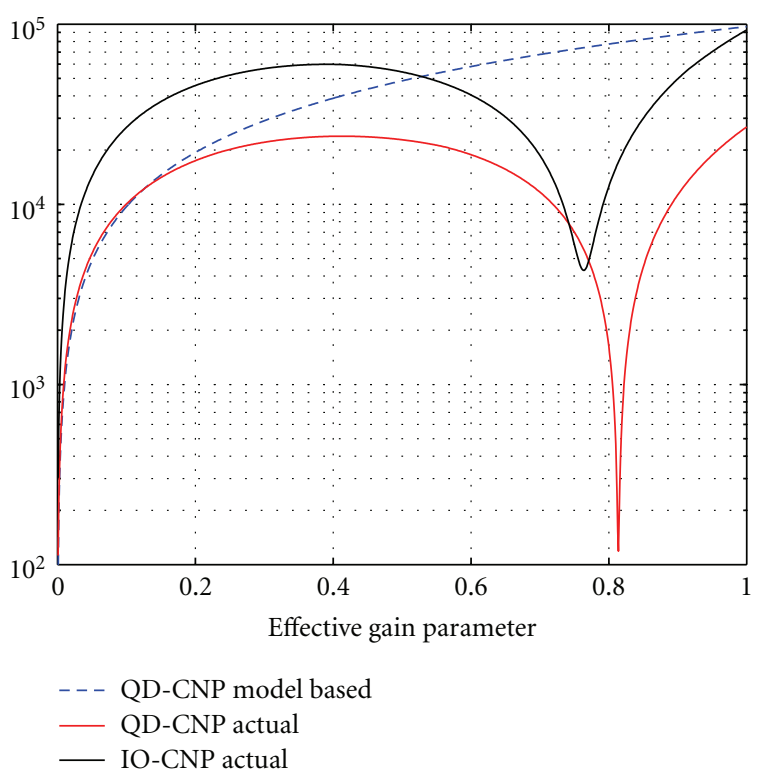

FIGURE 6: The core-shell model required gain values (blue) and the actual required gain values of the QD-CNP (red) and IO-CNP (black) designs $\left(\right.$ in $\mathrm{cm}^{-1}$ ).

However, the field overlap in the gain region is strong and is caused, in part, by an increase in the incident power flow into the gain region. This strong coupling of the incident field to the gain region leads to a large scattering cross-section $Q_{\text {scat }}$, which is in turn effectively related to the enhancement of the gain value by the amount $\sqrt{Q_{\text {scat }}}$, that is, the gain enhancement factor [6]. This enhanced coupling to the gain region thus leads to a reduction of the actual gain values required to achieve the results shown in Figure 4 . They are given in Figure 6, where the model-based gain value (blue) and the actual gain values of the QD-CNP (red) and IOCNP (black) designs are plotted versus the QD gain scale factor. The largest gain enhancement factor for the QD-CNP and IO-CNP configurations are, respectively, $\approx 287$ and $\approx 17$. We again note that the optimized configurations require less than the peak gain value to achieve their resonant states. Moreover, the tolerance of the gain scale factor (i.e., the widths of the resonances in Figure 6) to achieve a significant, high gain enhancement factor provides further tolerances in practice to potential fabrication and experimental errors.

We note that the IO-CNP design has a similar, but weaker behavior. It simply does not possess as large a gain enhancement factor. This is due to the QD layer being the outermost one. In the QD-CNP case, the QD layer is within the cavity formed by the silica core and the metal shell. Thus, the coupling of the large fields in the core to the SPPs created in the Ag shell at the Ag-QD (i.e., an $\epsilon<0$, $\epsilon>0$ ) interface provides a much larger feedback mechanism through the QD gain layer. This feedback is much smaller in the IO-CNP case because it occurs between the outside vacuum region and the metal shell. These interpretations are supported by earlier 3-layer observations [6] that included an exterior metal coating to the basic IO-CNP configuration that substantially improved its performance.

\section{Conclusions}

With an enhancement factor of $\approx 287$, the actual required gain value in the $\mathrm{QD}-\mathrm{CNP}$ configuration might be achieved with only 1 QD instead of the predicted 282 QDs that would make up an entire layer. In reality, the actual effectiveness of coupling energy into each QD would be lower than these ideal values. Nevertheless, the large enhancement factors observed indicate that designs using only a few QDs may be possible and that QD-QD coupling effects, while out of the scope of this study, can quite possibly be ignored due to the drastically reduced QD requirements. Also, the reduction of quenching effects seen with highly resonant plasmonic structures [14-20] suggests that QDs placed near the metal layer may not suffer from quenching effects, but rather could be further enhanced by the plasmonic resonance. These results further suggest that QDs may be a very realistic gain medium for active CNP designs and that QD-CNPs would be viable candidates for a variety of nanoamplifier applications. While the Mie theory analysis is limited in its scope, it has been used to describe the experimentally observed spectra of active NPs [4] and provides a good foundation for analyzing the effects of the resonant CNPs. The inclusion of off-theshelf QD geometry and performance characteristics in this modeling added engineering realism to its outcomes. Future work includes experimentally encapsulating QDs in these CNP structures to measure the predicted enhancements in their emissions and, hence, to verify the theory and modeling.

\section{Acknowledgment}

This work was supported in part by ONR Contract no. H940030920902 and NSF Contract no. ECCS-0823864.

\section{References}

[1] J. A. Gordon and R. W. Ziolkowski, "The design and simulated performance of a coated nano-particle laser," Optics Express, vol. 15, no. 5, pp. 2622-2653, 2007.

[2] M. I. Stockman, "The spaser as a nanoscale quantum generator and ultrafast amplifier," Journal of Optics A, vol. 12, no. 2, Article ID 024004, 2010.

[3] S. Arslanagić and R. W. Ziolkowski, "Active coated nanoparticle excited by an arbitrarily located electric Hertzian dipole-resonance and transparency effects," Journal of Optics A, vol. 12, no. 2, Article ID 024014, 2010.

[4] M. A. Noginov, G. Zhu, A. M. Belgrave et al., "Demonstration of a spaser-based nanolaser," Nature, vol. 460, no. 7259, pp. 1110-1112, 2009.

[5] Y. Sivan, S. Xiao, U. K. Chettiar, A. V. Kildishev, and V. M. Shalaev, "Frequency-domain simulations of a negativeindex material with embedded gain," Optics Express, vol. 17, no. 26, pp. 24060-24074, 2009.

[6] S. D. Campbell and R. W. Ziolkowski, "Impact of strong localization of the incident power density on the nanoamplifier characteristics of active coated nano-particles," Optics Communications, vol. 285, no. 16, pp. 3341-3352, 2012.

[7] P. Holmström, L. Thyĺn, and A. Bratkovsky, "Dielectric function of quantum dots in the strong confinement regime," 
Journal of Applied Physics, vol. 107, no. 6, Article ID 064307, 2010.

[8] http://www.sigmaaldrich.com/materials-science/nanomaterials/lumidots.html.

[9] B. Jensen and A. Torabi, "Refractive index of hexagonal II-VI compounds CdSe, CdS, and $\mathrm{CdSe}_{x} \mathrm{~S}_{1-x}$," Journal of the Optical Society of America B, vol. 3, pp. 857-863, 1986.

[10] http://www.dow.com/assets/attachments/business/gt/infrared _materials/cleartran/tds/cleartran.pdf.

[11] A. L. Aden and M. Kerker, "Scattering of electromagnetic waves from two concentric spheres," Journal of Applied Physics, vol. 22, no. 10, pp. 1242-1246, 1951.

[12] P. B. Johnson and R. W. Christy, "Optical constants of the noble metals," Physical Review B, vol. 6, no. 12, pp. 4370-4379, 1972.

[13] M. Klopfer and R. K. Jain, "Plasmonic quantum dots for nonlinear optical applications," Optical Materials Express, vol. 1, no. 7, pp. 1353-1366, 2011.

[14] W. C. H. Choy, X. W. Chen, S. L. He, and P. C. Chui, "The Purcell effect of silver nanoshell on the fluorescence of nanoparticles," in Proceedings of the Asia Optical Fiber Communication and Optoelectronic Exposition and Conference (AOE '07), pp. 81-83, October 2007.

[15] A. Neogi, H. Morkoç, T. Kuroda, and A. Tackeuchi, "Coupling of spontaneous emission from GaN-AlN quantum dots into silver surface plasmons," Optics Letters, vol. 30, no. 1, pp. 9395, 2005.

[16] J. H. Song, T. Atay, S. Shi, H. Urabe, and A. V. Nurmikko, "Large enhancement of fluorescence efficiency from CdSe/ZnS quantum dots induced by resonant coupling to spatially controlled surface plasmons," Nano Letters, vol. 5, no. 8, pp. 1557-1561, 2005.

[17] F. Tam, G. P. Goodrich, B. R. Johnson, and N. J. Halas, "Plasmonic enhancement of molecular fluorescence," Nano Letters, vol. 7, no. 2, pp. 496-501, 2007.

[18] Y. Jin and X. Gao, "Plasmonic fluorescent quantum dots," Nature Nanotechnology, vol. 4, no. 9, pp. 571-576, 2009.

[19] M. A. Noginov, H. Li, Y. A. Barnakov et al., "Controlling spontaneous emission with metamaterials," Optics Letters, vol. 35, no. 11, pp. 1863-1865, 2010.

[20] V. N. Pustovit and T. V. Shahbazyan, "Fluorescence quenching near small metal nanoparticles," Journal of Chemical Physics, vol. 136, no. 20, Article ID 204701, 2012. 

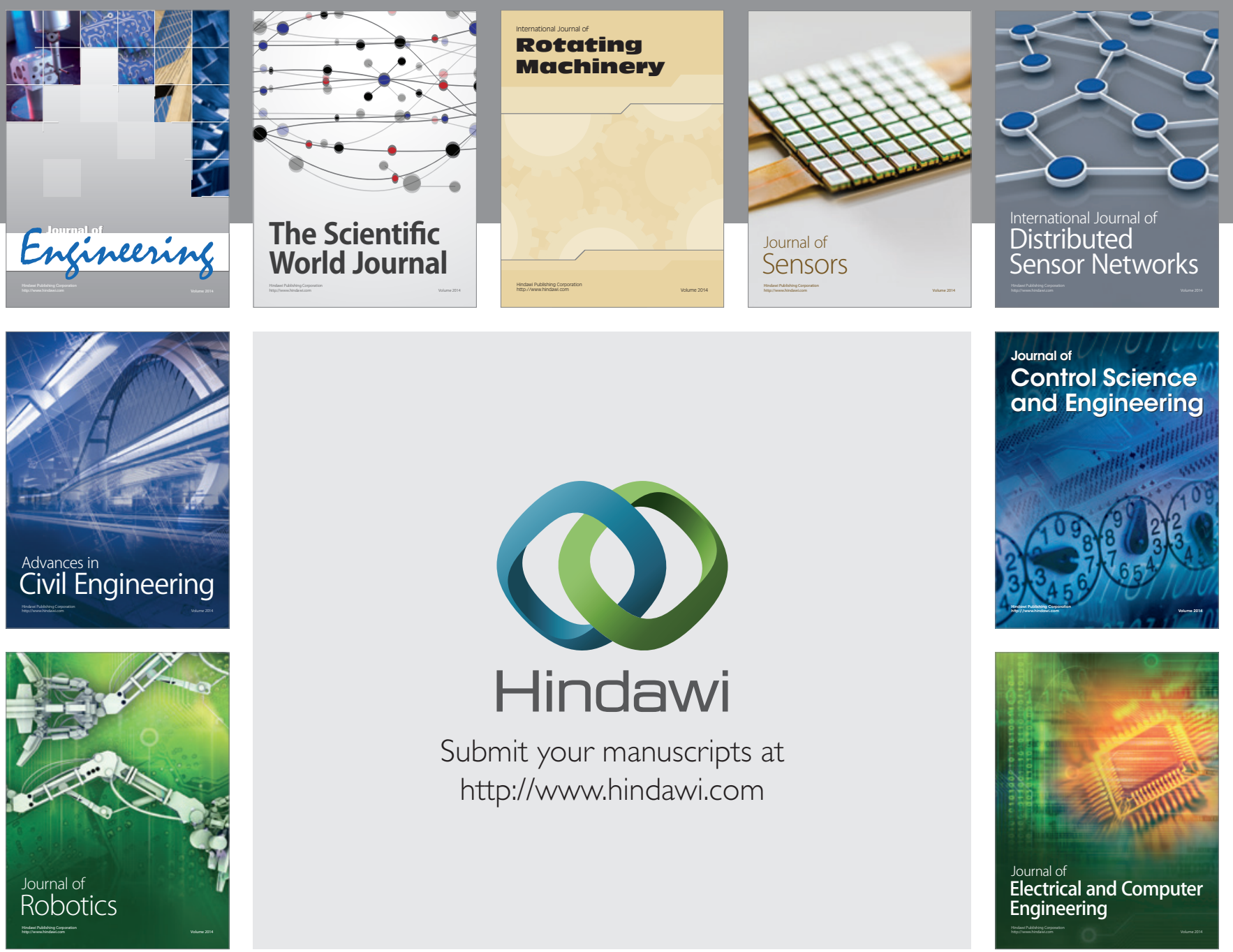

Submit your manuscripts at

http://www.hindawi.com
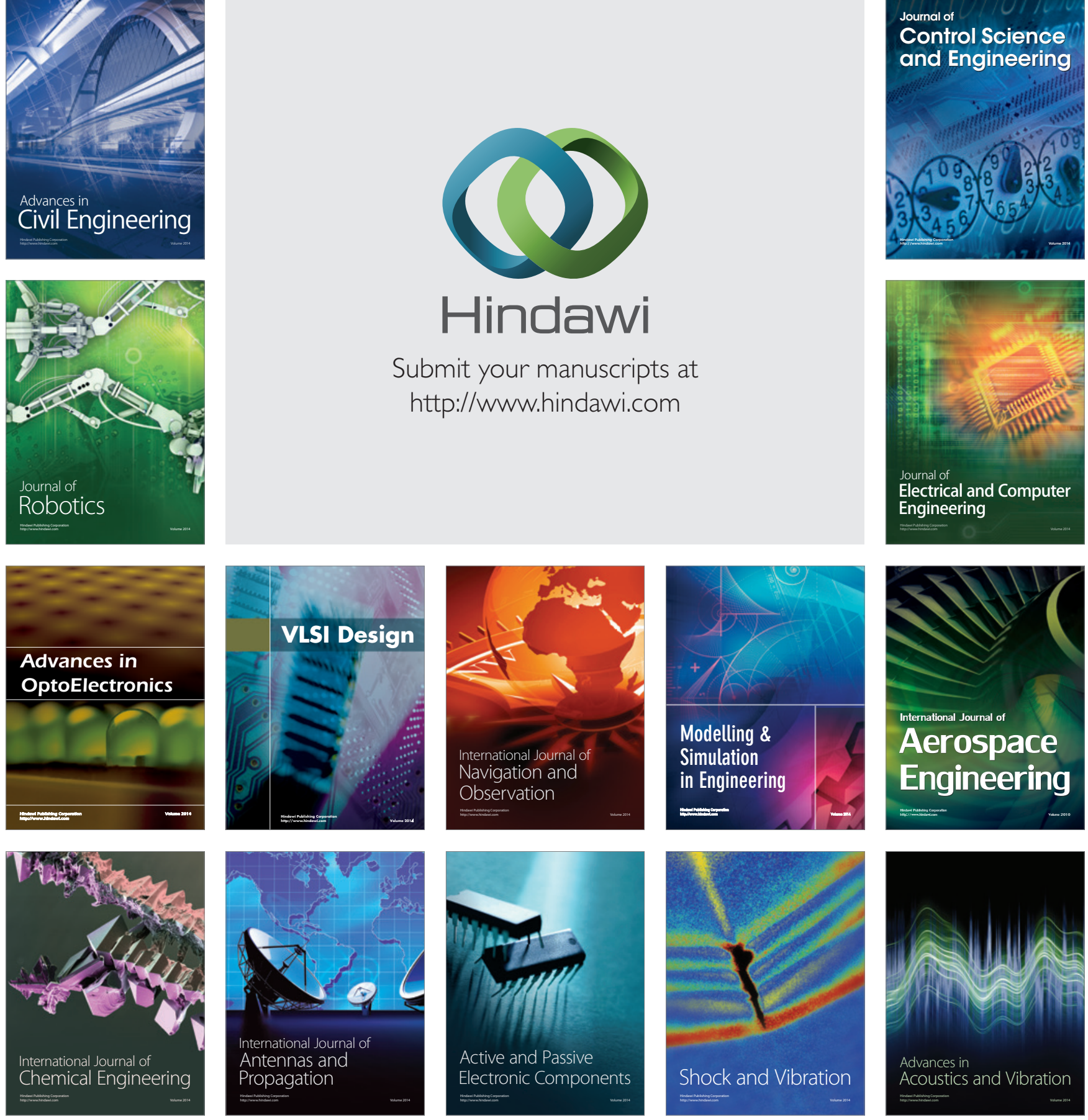